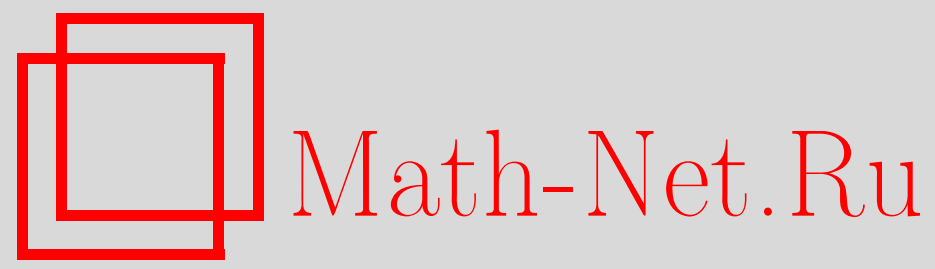

В. В. Козлов, Эйлер и математические методы механики, Совр. пробл. матем., 2008, выпуск 11, 39-70

DOI: https://doi.org/10.4213/spm22

Использование Общероссийского математического портала MathNet.Ru подразумевает, что вы прочитали и согласны с пользовательским соглашением

http://www . mathnet.ru/rus/agreement

Параметры загрузки:

IP : 18.234 .156 .22

26 апреля 2023 г., 17:20:27

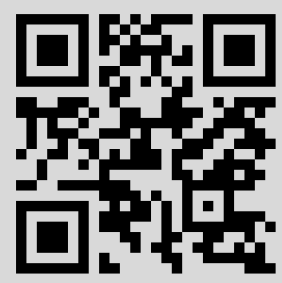




\title{
Эйлер и математические методы механики
}

\author{
В. В. Козлов
}

Эта статья - очерки о жизни и о достижениях Леонарда Эйлера в области теоретических проблем механики. Обсуждается ряд тем, связанных с развитием идей и методов Эйлера (расходящиеся ряды и асимптотики решений нелинейных дифференциальных уравнений, гидродинамика идеальной жидкости и гамильтоновы системы, вихревая теория систем на группах Ли с левоинвариантной кинетической энергией, энергетические критерии устойчивости, задача Эйлера двух гравитирующих центров в искривленных пространствах).

\section{1. Введение}

Эта работа посвящена творчеству величайшего математика Леонарда Эйлера. Его наследие в математике и ее приложениях столь грандиозно, что еще не в полной мере осознано и систематизировано. Жизнь Эйлера - яркий пример интернациональности науки (и математики в особенности). Он родился в Швейцарии, там же получил образование и раскрыл свое математическое дарование под руководством знаменитого Иоганна Бернулли, который в свою очередь был прямым учеником самого́ Лейбница. Затем 19-летним молодым человеком по совету своих друзей Николая и Даниила Бернулли он прибыл в Санкт-Петербург, чтобы получить стабильное место работы с хорошей заработной платой. Как заметил Кондорсе, "они употребили столько же усилий для того, чтобы приблизить к себе своего страшного соперника, сколько их употребляют обыкновенные люди для удаления такового". Мы увидим далее, что, действительно, Леонард Эйлер и Даниил Бернулли часто соперничали, занимаясь параллельно одними и теми же теоретическими проблемами механики.

В наше время молодые российские математики с теми же целями часто уезжают в Европу и Америку. Во времена Эйлера все было наоборот. Конечно, тогда молодой Петербург еще мало походил на европейскую столицу: Эйлер и Даниил Бернулли, который

(C) Козлов В. В., 2008 
в то время уже работал в России, фактически были ровесниками города. В своих письмах Даниил жаловался отцу Иоганну Бернулли на холод и скуку Петербурга. Соглашаясь с этим, Иоганн, однако, повторял, что в Европе нет больше такого места, где так уважают науку и так хорошо за нее платят.

Интересно отметить, что вначале Эйлер был зачислен на должность адъюнкта по кафедре физиологии, поскольку все места по математике и физике были заняты. Он даже всерьез штудировал анатомию и медицину в своем родном Базеле перед поездкой в Россию. Впрочем вскоре его перевели сначала на кафедру физики, а в 1733 г. он занял место своего друга Даниила Бернулли кафедру математики. Д. Бернулли вернулся в Базель из-за расстроенного здоровья, а его старший брат Николай умер на следующий год по приезде в Петербург. Кстати сказать, надпись под бюстом Эйлера, установленным в здании Президиума Российской академии наук, продолжает утверждать, что Леонард Эйлер физиолог, физик и (только потом!) математик.

В общей сложности Эйлер проработал в Петербурге 31 год и скончался здесь 76 лет от роду. Когда в 1740-х годах общая обстановка в России оказалась неблагоприятной для науки, Эйлер принял приглашение прусского императора Фридриха II и проработал директором математического класса Берлинской академии наук 25 лет. Стоит подчеркнуть, что будучи в Берлине, Эйлер продолжал активно сотрудничать с Петербургской академией, регулярно публикуя свои многочисленные статьи по математике и механике в ее "Комментариях". Посмертными трактатами Л. Эйлера еще многие годы заполнялись журналы, которые издавались Петербургской академией наук.

Эйлер был самым авторитетным и уважаемым ученым в России. Можно напомнить известный случай, когда княгиня Дашкова, назначенная директором Петербургской академии, попросила именно Эйлера сопровождать ее при первом посещении Академии (хотя он в то время не ладил с академическим начальством и даже не посещал академических конференций).

Рассказывают, что Эйлер однажды решился попросить у Екатерины II генеральский чин тайного советника (как академик он был действительным статским советником, что соответствовало армейскому чину полковника). Перед ним был пример Христиана Гольдбаха, который состоял на государственной службе России и работал криптографом в Министерстве иностранных дел 
в Москве. Эйлер вел с ним оживленную переписку, в которой, в частности, обсуждались ставшие знаменитыми гипотезы из аддитивной теории чисел. Однако Екатерина отшутилась: "Господин академик! Генералов у меня много, а Эйлер - всего один!"

\section{2. Расходящиеся ряды и обратная теорема Лагранжа-Дирихле}

Бытует мнение (сформированное не только трудами по истории науки), что математики того времени (в частности, и Эйлер) часто путались в элементарных вопросах. Например, не отличали дифференцируемые функции от непрерывных, мешали в одну кучу сходящиеся и расходящиеся ряды и т. д. Конечно, тогда в математике было меньше схоластики, не принято было давать точные определения и многие математические структуры не были выделены и четко очерчены. Однако следует предостеречь от поверхностного взгляда на эти вопросы. Было бы верхом наивности утверждать, что Әйлер не понимал различия между сходящимися и расходящимися рядами. Как точно подметил Харди, в этих вопросах математикам того времени не хватало техники. Им не был еще известен критерий сходимости Коши и поэтому было затруднительным сделать вывод о сходимости ряда без попытки вычисления его суммы. В оперировании с расходящимися рядами точка зрения Эйлера существенно более гибкая и более глубокая, чем это излагается в стандартных университетских курсах анализа. Когда выпускнику университета, хорошо усвоившему такой курс, встретится расходящийся ряд, то он его отбросит как нечто запрещенное и даже несуществующее. Между тем полезно попытаться найти сумму этого расходящегося ряда, применив подходящий метод суммирования. Зачастую такой подход решает дело. Ведь вся эргодическая теория по существу построена на замене обычной сходимости на сходимость по Чезаро (средних арифметических).

Вот один из результатов Эйлера:

$$
1-1 !+2 !-3 !+4 !-\cdots=0.5963 \ldots
$$

Он получил эту формулу несколькими различными способами.

Однако, по мнению акад. А. Н. Крылова,

"Все эти формулы в математике совершенно бесполезны. . ." 
В дальнейшем последователи Эйлера, преклоняясь перед его авторитетом, как бы старались приумножить число этих нелепых равенств, зачастую забыв об условном их смысле, придаваемом Эйлером, и таким образом создали тот скандал в математике, который продолжался 75 лет. . .

Скандал этот был прекращен Гауссом, Коши и Абелем, изгнавшими из строгой математики пользование рядами без исследования их сходимости" [1].

Такая консервативная точка зрения, конечно, малопродуктивна. Когда А. Н. Крылов писал эти строки, теория суммирования расходящихся рядов была уже сильно продвинута (см. фундаментальную монографию Г. Харди [2], в которой много места уделяется истории вопроса и, в частности, вкладу Эйлера в развитие этой теории).

Вот как можно посмотреть на формулу Эйлера (1). Рассмотрим простую нелинейную систему дифференциальных уравнений на плоскости $\mathbb{R}^{2}=\{x, y\}$ :

$$
\dot{x}=x-y, \quad \dot{y}=-y^{2} .
$$

Будем искать ее решения, которые асимптотически стремятся к положению равновесия $x=y=0$ при $t \rightarrow+\infty$. Второе уравнение (2) имеет очевидное асимптотическое решение $y(t)=1 / t$. Подставим это решение в первое уравнение и найдем формальное решение полученного линейного неоднородного уравнения в виде ряда по обратным степеням $t$ :

$$
x=\sum_{k=1}^{\infty}(-1)^{k+1} \frac{(k-1) !}{t^{k}} .
$$

При $t=1$ получаем ряд Эйлера. С другой стороны, система (2) имеет стремящееся к нулю решение

$$
x(t)=e^{t} \int_{t}^{\infty} \frac{e^{-u}}{u} d u, \quad y(t)=\frac{1}{t},
$$

причем расходящийся ряд (3) является асимптотическим представлением функции $x(t)$ при $t \rightarrow+\infty$ (в чем легко убедиться последовательным интегрированием по частям). Подставляя $t=1$, получим сумму ряда Эйлера:

$$
e \int_{1}^{\infty} \frac{e^{-u}}{u} d u=0.5963 \ldots
$$


Эти рассуждения только по форме отличаются от рассуждений самого́ Эйлера.

Вообще, пусть система дифференциальных уравнений в $\mathbb{R}^{n}=$ $\{x\}$

$$
\dot{x}=v(x), \quad v(0)=0
$$

с гладкой (бесконечно дифференцируемой) правой частью допускает формальное решение в виде ряда

$$
\sum_{j=1}^{\infty} \frac{x^{(j)}}{\left(t^{\mu}\right)^{j}}, \quad x^{(j)} \in \mathbb{R}^{n},
$$

где $\mu$ - некоторая положительная постоянная (для решения (3) $\mu=1)$. Тогда это уравнение имеет настоящее решение $t \mapsto x(t)$ (быть может, и не одно) такое, что

1) $x(t) \rightarrow 0$ при $t \rightarrow \infty$,

2) степенной ряд (5) будет для него асимптотическим:

$$
x(t)-\sum_{j=1}^{N} \frac{x^{(j)}}{\left(t^{\mu}\right)^{j}}=O\left(\frac{1}{t^{(N+1) j}}\right), \quad t \rightarrow \infty .
$$

Эта замечательная теорема доказана А.Н. Кузнецовым в 1972 г. [3]. Думаю, что Эйлер был бы доволен таким результатом. Впоследствии (по моему предложению) А. Н. Кузнецов обобщил эту теорему на случай, когда коэффициенты формального ряда (5) полиномиально зависят от логарифмов $t$, а также распространил ее на более общие шкалы сравнения [4].

С сожалением (и удивлением) приходится констатировать, что теория Кузнецова малоизвестна (особенно за рубежом). Например, в недавней книге Ж.-П. Рамиса [5], посвященной асимптотическому анализу решений дифференциальных уравнений, она даже не упоминается. Вместо нее фигурирует более слабое утверждение, к тому же полученное позже.

Приведу один пример из этого круга идей, связанный с проблемой обращения теоремы Лагранжа-Дирихле об устойчивости, поставленной А.М. Ляпуновым еще в 1897 году. Теорема Лагранжа-Дирихле утверждает, что если в положении равновесия потенциальная энергия системы имеет строгий локальный минимум, то равновесие устойчиво. Этот энергетический критерий устойчивости широко используется и для исследования устойчивости состояний равновесия систем с распределенными 
параметрами. В связи с этим важно указать необходимые условия устойчивости.

Движение системы в потенциальном силовом поле описывается уравнением Лагранжа

$$
\frac{d}{d t} \frac{\partial T}{\partial \dot{x}}-\frac{\partial T}{\partial x}=-\frac{\partial V}{\partial x}, \quad x \in \mathbb{R}^{n},
$$

где

$$
T=\frac{1}{2} \sum g_{i j}(x) \dot{x}_{i} \dot{x}_{j}
$$

- положительно определенная квадратичная форма по скоростям $\left(\dot{x}_{1}, \ldots, \dot{x}_{n}\right)=\dot{x}$ (кинетическая энергия системы), а $V: \mathbb{R}^{n} \rightarrow \mathbb{R}-$ потенциальная энергия. Пусть $d V(0)=0$. Тогда $x=0$ - положение равновесия. Пусть функции $g_{i j}$ и $V$ бесконечно дифференцируемы и

$$
V=V(0)+V_{2}+V_{m}+V_{m+1}+\cdots
$$

- разложение Маклорена потенциальной энергии в ряд по однородным формам $\left(V_{k}(\lambda x)=\lambda^{k} V_{k}(x)\right)$. Так как $x=0$ - положение равновесия, то в этом разложении отсутствуют слагаемые, линейные по $x_{1}, \ldots, x_{n}$.

В типичной ситуации, конечно, $m=3$. Однако для четных потенциалов $m=4$.

Как показал Ляпунов, если $V_{2}$ не имеет минимума в точке $x=0$, то это равновесие неустойчиво (причем экспоненциально). Поэтому рассмотрим случай, когда $V_{2} \geqslant 0$. Если квадратичная форма $V_{2}$ положительно определена, то равновесие $x=0$, наоборот, устойчиво (теорема Лагранжа-Дирихле). Так что полезно рассмотреть плоскость

$$
\Pi=\left\{x \in \mathbb{R}^{n}: V_{2}(x)=0\right\}
$$

и предположить, что $\operatorname{dim} \Pi \geqslant 1$. Пусть $W_{m}-$ ограничение однородной формы $V_{m}$ на П. Справедлива

Теорема 1 [6]. Если $W_{m}$ не имеет минимума в точке $x=0$, то равновесие неустойчиво.

В этом случае можно указать формальное решение уравнения (6) в виде ряда (5), где $\mu=2 /(m-2)$. При четных $m \geqslant 4$ коэффициенты $x^{(j)}$ полиномиально зависят еще от $\ln t$. Если $V_{2} \not \equiv 0$, то ряды (5), как правило, расходятся даже в предположении аналитичности функций $g_{i j}$ и $V$. Однако (по теореме Кузнецова [4]) 
уравнение (6) имеет решение $t \mapsto x(t)$, которое стремится к положению равновесия $x=0$ при $t \rightarrow+\infty$. Так как уравнение (6) не меняется при обращении времени $t \mapsto-t$, то $t \mapsto x(-t)$ будет решением, асимптотически исходящим из точки $x=0$. В частности, равновесие $x=0$ неустойчиво, причем эта неустойчивость имеет степенной характер.

Отметим, что теорема 1 опубликована на три года раньше общей теоремы Кузнецова, нужной для ее доказательства. Фактически теорема Кузнецова с логарифмами была анонсирована в работе [6].

Если в разложении (7) отсутствуют квадратичные слагаемые $\left(V_{2}=0\right)$, то в аналитическом случае формальный ряд (5) сходится при достаточно больших значениях $t$ [7]. Этот факт установлен даже в более общем случае, когда $x$-это точки гильбертова пространства. Если $V_{2} \not \equiv 0$ и $V_{2}$ не имеет минимума в точке $x=0$, то равновесие бесконечномерной системы также неустойчиво. Здесь асимптотическое решение следует искать в виде ряда по степеням $\exp (-\lambda t), \lambda>0$, который сходится при больших $t$. Эти результаты (вместе с теоремой Лагранжа-Дирихле) дают надежную основу для исследования устойчивости состояний равновесия систем с распределенными параметрами. Было бы интересным распространить теорему 1 на бесконечномерный случай. Это дало бы возможность доказать неустойчивость равновесия в точках бифуркации, когда второй дифференциал потенциальной энергии теряет положительную определенность.

Систематическое изложение затронутого круга вопросов можно найти в книге [8].

\section{3. Эйлер и механика}

Обратимся теперь к работам Леонарда Эйлера собственно по механике. Эта тема представляется совершенно необъятной, особенно с учетом его многочисленных прикладных исследований. Поэтому мы сосредоточимся на чисто теоретических работах Эйлера, посвященных математическим аспектам механики.

Сначала обсудим достижения Л. Эйлера в аналитической динамике.

А. Эйлер ввел и систематически использовал так называемые естественные уравнения движения материальной точки, когда уравнение Ньютона $m \ddot{r}=F$ проектируется на оси подвижного 
репера Френе. Эта идея потом была им использована в динамике твердого тела. Любопытно отметить, что более простая запись уравнений движения в неподвижном репере появилась позже в работах Маклорена.

Эйлер открыл, что в отсутствие внешних сил траектории материальной частицы, движущейся по гладкой регулярной поверхности, совпадают с геодезическими линиями этой поверхности.

Эйлер (одновременно с Д. Бернулли) ввел в механику момент количества движения системы частиц и нашел фундаментальную теорему о его изменении. В этой теореме фигурирует момент силы, известный до Эйлера и широко использовавшийся в задачах статики.

Эта теорема дала возможность Эйлеру вывести уравнения вращения твердого тела

$$
I \dot{\omega}+\omega \times I \omega=M .
$$

Здесь $\omega$ - угловая скорость тела в подвижной системе отсчета (связанной с телом), $I$ - оператор (тензор) инерции, а $M-$ суммарный момент сил, действующих на тело. При выводе этого уравнения использовалась открытая Эйлером теорема о распределении скоростей в движущемся твердом теле, а также теория моментов инерции.

Рассматривая изменение момента инерции тела в зависимости от направления прямой, Эйлер доказал существование трех взаимно ортогональных прямых (главных осей инерции), обладающих экстремальными значениями моментов инерции. В этих осях симметричный оператор $I$ приводится к диагональному виду. По существу это первые теоремы линейной алгебры, относящиеся к преобразованиям квадратичных форм.

Эйлер ввел координаты, определяющие ориентацию тела (углы Эйлера) и представил угловую скорость через эти углы и их производные по времени (кинематические формулы Эйлера). Эти соотношения вместе с динамическими уравнениями (8) составляют замкнутую систему дифференциальных уравнений, описывающую вращение твердого тела вокруг неподвижной точки под действием заданных сил. Эйлер проинтегрировал свои уравнения в простом, но важном случае, когда $M=0$ (волчок Эйлера).

Безусловно, Леонард Эйлер - основатель динамики твердого тела, имеющей кроме всего прочего и большое прикладное значение. 
Эйлер сформулировал и обосновал принцип наименьшего действия. Вначале этот вариационный принцип был установлен для движения одной материальной точки в потенциальном силовом поле, затем Лагранж его распространил на систему взаимодействующих частиц, а впоследствии Якоби придал ему привычную сейчас форму. Любопытно отметить, что Эйлер и Лагранж классики вариационного исчисления - не заметили более простого и фундаментального вариационного принципа Гамильтона, из которого принцип наименьшего действия выводится как следствие. Вариационные принципы играют существенную роль не только в аналитической механике, но и в математической и теоретической физике.

История открытия принципа наименьшего действия весьма поучительна. Я приведу ее в очень живом изложении Якоби (с некоторыми сокращениями) [9].

"Рождение этого принципа было связано с большим литературным гамом, вряд ли когда еще поднимали такую суматоху. Открытие этого принципа приписывается бывшему президенту Берлинской Академии Мопертюи, который хотя и был умным человеком, и с некоторыми идеями, но мало учился и в открытом недавно исчислении бесконечно малых разбирался не так хорошо, как великие математики того времени...

... в 1744 г. Мопертюи обставил с большой помпой свое сообщение Парижской АН, в котором говорилось, что математика до этого времени повсюду использовалась в самых незначительных целях, а уж он-то найдет ей достойное применение, выведя все законы природы непосредственно из божьих атрибутов. При этом под названием "принцип наименьшего действия" он формулирует принцип, из-за которого ни одна собака с печи не слезет, в то время как эйлеровский охватывает всю механику...

Мопертюи обнародовал свою теорему незадолго до отъезда из Парижа в Берлин, чтобы стать там президентом Академии, а затем написал о ней второй трактат в берлинских Memoiren. Тон, которым он был написан, естественно, вскоре встретил возражения...

... но особенно возмущался некий математик Кениг. Этот последний был странным человеком: в юности он вместе с тем же Мопертюи и Клеро учился у старого Иоганна Бернулли в Базеле, а затем втянулся в демагогические волнения. ., направлен- 
ные против аристократических тенденций, и был изгнан из своего родного города Берна. . .

И вот он напал на Мопертюи в Actis Eruditorum, одной очень известной газете... K этой статье Кениг присоединил отрывок из письма Лейбница..., в котором Лейбниц дает понять, что он подробно изучил действие, и что оно всегда будет минимумом. . .

Разразился крупный скандал, и от Кенига потребовали оригинал письма. Но у того была лишь копия, доставшаяся ему от некоего Хинцеля, повешенного при упомянутых волнениях в Цюрихе... Позднее Кениг обнародовал письмо целиком, оно содержит немало интересного, однако, кажется, как будто он выдумал добавление о минимуме действия лишь, чтобы позлить Мопертюи. Эйлеру поручили написать сочинение и вывести Кенига на чистую воду, и Берлинская Академия торжественно подтвердила разоблачение обмана на одном из своих заседаний. . .

... Эйлер повел себя так, что принцип Мопертюи он все время подменял своим принципом наименьшего действия, при защите отождествил оба и сказал, что уж если доказанный им для отдельных случаев принцип так важен, то что можно сказать о принципе Мопертюи, которого он выставил открывателем всех законов механики и физики".

В. Леонард Эйлер - один из творцов гидродинамики. Уравнения движения жидкости можно представить в двух различных формах: или мы исследуем скорость, давление и плотность во всех точках течения, или же мы исследуем историю отдельной частицы жидкости. Уравнения, которые получаются этими двумя способами, обычно называются эйлеровой и лагранжевой формами уравнений гидродинамики (хотя обе принадлежат Эйлеру).

Наиболее популярны уравнения Эйлера, описывающие движение идеальной жидкости:

$$
\rho\left(\frac{\partial v}{\partial t}+\frac{\partial v}{\partial x} v\right)=-\frac{\partial p}{\partial x}-\rho \frac{\partial V}{\partial x} .
$$

Здесь $x=\left(x_{1}, x_{2}, x_{3}\right)$ - точка трехмерного евклидова пространства, $v(x, t)$ - поле скоростей частиц жидкости, $\rho(x, t)$ - плотность, $p(x, t)$ - давление, $V(x, t)$ - плотность потенциальной энергии массовых сил. К трем скалярным уравнениям (9) добавляется урав- 
нение неразрывности

$$
\frac{\partial \rho}{\partial t}+\operatorname{div}(\rho v)=0
$$

которое выражает закон сохранения массы движущегося объема жидкости. Оно также получено Эйлером. Обычно рассматривают баротропную жидкость, когда давление и плотность связаны некоторым функциональным соотношением. Это дополнительное предположение замыкает систему нелинейных дифференциальных уравнений (9) и (10). Основные гидродинамические результаты Эйлера опубликованы в 1755 г. (Берлин) и 1759 г. (С.Петербург).

Для баротропной жидкости уравнение (9) можно представить в виде уравнения Ламба

$$
\frac{\partial v}{\partial t}+(\operatorname{rot} v) \times v=-\frac{\partial h}{\partial x},
$$

где $h=v^{2} / 2+\mathscr{P}+V$, а $\mathscr{P}-$ функция давления $\left(\rho^{-1} d p=d \mathscr{P}\right)$. Функция $h$ называется функцией Бернулли. В стационарном случае $h$ постоянна на линиях тока (теорема Д. Бернулли). Этот факт был положен Даниилом Бернулли в основу своей "Гидродинамики", опубликованной в 1738 г.

Стационарные течения идеальной жидкости хорошо изучены. Во-первых, векторные поля $v$ и $\operatorname{rot} v$ касаются поверхностей Бернулли

$$
B_{c}=\{x: h(x)=c\} .
$$

Эти поверхности регулярны, если поле $v$ неколлинеарно своему ротору. Более того, поля

$$
v \text { и }(\operatorname{rot} v) / \rho
$$

коммутируют. Этот факт отмечен В. И. Арнольдом [10] для однородной несжимаемой жидкости ( $\rho=$ const) и автором [11] в общем случае. Следовательно, если поверхности Бернулли ограничены, то они диффеоморфны двумерным торам, причем частицы жидкости движутся условно-периодически. Если же поля $v$ и rot $v$ коллинеарны, то течение в замкнутой области, как правило, будет хаотическим.

C. В математической теории упругости Эйлер вывел дифференциальное уравнение для упругой кривой (эластики) и дал классификацию ее форм равновесий (1744 г.). 
Определил наименьшую высоту колонны, при которой она может изогнуться под действием своего собственного веса или приложенной извне нагрузки. Лагранж следовал его теории и применил ее к определению наиболее надежной формы колонн. Эти работы - первые исследования по устойчивости упругих систем, в которых ключевую роль играет энергетический критерий устойчивости (для систем с конечным числом степеней свободы - это упоминавшаяся выше теорема Лагранжа-Дирихле).

Наконец, вслед за Тейлором, Эйлер получил дифференциальное уравнение поперечных колебаний стержня, нашел собственные функции и вывел уравнения частот для различных краевых условий. Параллельно с ним эти результаты получил и Даниил Бернулли.

D. Работы Эйлера по небесной механике и астрономии носят, в основном, прикладной характер (например, его теория движения Луны, по которой были составлены практические таблицы). Однако имеются результаты Эйлера по задаче трех тел, которые вошли в "золотой фонд" небесной механики. Во-первых, это частные движения трех гравитирующих тел, которые все время остаются на одной подвижной прямой. Далее, Эйлер проинтегрировал плоскую задачу двух неподвижных центров, когда движущаяся частица притягивается двумя неподвижными точками. Если переносить один из притягивающих центров в бесконечность и согласованным образом увеличивать его массу, то получим важную предельную интегрируемую задачу Кеплера в однородном силовом поле. Эта задача имеет существенное значение в квантовой механике: атом водорода в однородном электрическом поле. С ней связан "эффект Штарка" - расщепление спектральных линий атомов в электрическом поле.

Впоследствии Лагранж показал интегрируемость задачи двух центров в пространстве, а также обобщил ее на случай, когда движущаяся точка притягивается (или отталкивается) упругой силой, направленной к точке, лежащей посредине между центрами.

О научном стиле Эйлера удачно сказал Якоби в своих "Лекциях по динамике": "Работы Эйлера имеют вообще ту большую заслугу, что им везде приведены по возможности все случаи, в которых задачи могут быть решены полностью с помощью данных способов и средств. . . . как правило, когда удается к примерам Эй- 
лера присоединить какой-нибудь новый пример, то это является обогащением науки".

В связи с этим высказыванием упомянем замечательный результат С. В. Болотина о неинтегрируемости плоской задачи $n$ неподвижных центров при $n \geqslant 3$ [12].

Мы сейчас перекинем мостики к некоторым новым исследованиям в этих областях, где идеи и методы Эйлера имеют существенное значение.

\section{4. Гидродинамика гамильтоновых систем}

Гидродинамические уравнения Эйлера (9) возникают естественным образом при изучении инвариантных многообразий гамильтоновых систем, однозначно проектирующихся на конфигурационное пространство.

Пусть $M^{n}-n$-мерное конфигурационное многообразие механической системы с локальными координатами $x=\left(x_{1}, \ldots, x_{n}\right)$, $\Gamma=T^{*} M-$ фазовое пространство (кокасательное расслоение $M$ ), $y=\left(y_{1}, \ldots, y_{n}\right)-$ канонические импульсы в точке $x \in M$ (это ковекторы - элементы пространства $T_{x}^{*} M$, двойственного касательному пространству $\left.T_{x} M\right), H(x, y, t)$ - функция Гамильтона (заданная на $Г$ и зависящая, вообще говоря, от времени $t$ ). Эволюция определяется системой канонических уравнений Гамильтона

$$
\dot{x}_{j}=\frac{\partial H}{\partial y_{j}}, \quad \dot{y}_{j}=-\frac{\partial H}{\partial x_{j}}, \quad 1 \leqslant j \leqslant n .
$$

Пусть $\Sigma_{t}^{n}$ - многообразие в фазовом пространстве $Г$, однозначно проектирующееся на конфигурационное пространство $M$. В канонических координатах $x, y$ оно задается уравнениями

$$
y=u(x, t)
$$

где $u$ - некоторое ковекторное поле на $M$, зависящее, возможно, от времени. Свойство инвариантности многообразия $\Sigma_{t}$ означает следующее. Пусть $x(t), y(t)$ - решение уравнений Гамильтона с начальными данными

$$
x\left(t_{0}\right)=x_{0}, \quad y\left(t_{0}\right)=y_{0} .
$$

Если точка $\left(x_{0}, y_{0}\right)$ принадлежит $\Sigma_{t_{0}}$, то

$$
(x(t), y(t)) \in \Sigma_{t}
$$


для всех значений $t$.

Несложно проверить, что критерий инвариантности многообразия $\Sigma_{t}$ состоит в том, что поле (13) удовлетворяет уравнению

$$
\frac{\partial u}{\partial t}+(\operatorname{rot} u) v=-\frac{\partial h}{\partial x}
$$

где $\operatorname{rot} u=\partial u / \partial x-(\partial u / \partial x)^{T}-$ кососимметрическая $n \times n$-матрица (ротор ковекторного поля $u$ ),

$$
v(x, t)=\left.\frac{\partial H}{\partial y}\right|_{y=u(x, t)}
$$

- векторное поле на $M, h(x, t)=H(x, u(x, t), t)$ - функция на $M$, зависящая от времени $t$ как параметра.

Для "натурального" гамильтониана

$$
H=\frac{1}{2} \sum_{i, j=1}^{n} g_{i j}(x, t) y_{i} y_{j}+V\left(x_{1}, \ldots, x_{n}, t\right)
$$

уравнение (14) имеет следующий явный вид:

$$
\begin{aligned}
\frac{\partial u_{i}}{\partial t} & +\sum_{j, k} g_{j k}\left(\frac{\partial u_{i}}{\partial x_{j}}-\frac{\partial u_{j}}{\partial x_{i}}\right) u_{k} \\
& =-\frac{1}{2} \frac{\partial}{\partial x_{i}}\left(\sum_{j, k} g_{j k} u_{j} u_{k}\right)-\frac{\partial V}{\partial x_{i}}, \quad i=1, \ldots, n .
\end{aligned}
$$

Это - нелинейная система $n$ дифференциальных уравнений в частных производных первого порядка относительно $n$ неизвестных функций $u_{1}, \ldots, u_{n}$.

Пусть, например, $n=3$ и $g_{i j}=\delta_{i j}$ (символы Кронекера). Тогда $u=v$ и уравнения (15) будут иметь вид гидродинамических уравнений Эйлера, представленных в форме Ламба. Функцию $h$ будем называть функцией Бернулли.

Движения рассматриваемой системы, фазовые траектории которых лежат на $\Sigma$, находятся как решения системы обыкновенных дифференциальных уравнений на $M$ :

$$
\dot{x}=v(x, t), \quad x \in M .
$$

Эти соображения позволяют применить идеи и методы гидродинамики к изучению гамильтоновых систем [13]. С этой целью 
введем вихревые многообразия как естественное обобщение вихревых линий в классической гидродинамике. Собственные векторы матрицы $\operatorname{rot} u$ с нулевым собственным значением назовем вихревыми векторами. В каждой точке $x \in M$ в любой момент времени $t$ касательные вихревые векторы образуют линейное пространство. Возникает распределение касательных плоскостей на $M$. Можно показать (в предположении постоянства размерности плоскостей вихревых векторов), что это распределение интегрируемо. Интегральные многообразия этого распределения естественно назвать вихревыми многообразиями.

Оказывается, вихревые многообразия "вморожены" в поток системы дифференциальных уравнений (16) на $M$. Другими словами, преобразования из этого потока переводят вихревые многообразия в вихревые многообразия. Это - многомерный вариант классической теоремы Гельмгольца-Томсона из гидродинамики идеальной жидкости. Справедливо также следующее естественное обобщение теоремы Бернулли: в стационарном случае функция Бернулли $h$ постоянна как на линиях тока (интегральных кривых векторного поля $v$ на $M$ ), так и на вихревых многообразиях.

Рассмотрим потенциальное решение уравнения Ламба (14), когда $u=\partial S / \partial x$, где $S$ - функция от координат $x$ и времени $t$. В этом случае уравнение (14) эквивалентно соотношению

$$
\frac{\partial S}{\partial t}+H\left(x, \frac{\partial S}{\partial x}, t\right)=f(t)
$$

Тривиальная калибровка

$$
S \mapsto S-\int f(t) d t
$$

позволяет обнулить правую часть уравнения (17), которое превращается в известное уравнение Гамильтона-Якоби. В гидродинамике (17) называется интегралом Лагранжа-Коши.

Классический метод Якоби интегрирования дифференциальных уравнений Гамильтона (12) сводится к отысканию полного решения уравнения (17), невырожденно зависящего еще от $n$ параметров $c_{1}, \ldots, c_{n}$ :

$$
\operatorname{det}\left\|\frac{\partial^{2} S}{\partial c_{i} \partial x_{j}}\right\| \neq 0 .
$$


В [14] указано обобщение метода Якоби, основанное на поиске полного решения уравнений Ламба. Приведу простейший вариант теоремы об интегрируемости в стационарном случае при $n=3$. Пусть известно решение уравнения (14) $u(x, c), c=\left(c_{1}, c_{2}, c_{3}\right)$, такое, что

1) $\operatorname{rot} u \neq 0$,

2) $\left|\frac{\partial u}{\partial c}\right| \neq 0$

3) $d_{x} H(x, u(x, c)) \neq 0$.

Тогда уравнение Гамильтона интегрируется в квадратурах.

Это утверждение доказывается с помощью теории "последнего множителя" Эйлера-Якоби. Согласно Якоби, “. . . теория Эйлера по введению множителя в интегральное уравнение является одним из его самых замечательных открытий в интегральном исчислении".

Уравнение (14) появилось, по-видимому, впервые в вариационном исчислении как условие согласованности полей экстремалей (которые, как известно, описываются каноническими уравнениями Гамильтона). Правда, там обычно рассматриваются лишь самосопряженные (потенциальные) поля. И. С. Аржаных [15] обобщил уравнение Ламба на негамильтоновы системы (в частности, неголономные) и пытался распространить на них метод Гамильтона-Якоби. Однако до работы [13] уравнение (14) обычно не связывали с идеями гидродинамики. Систематическое изложение аналогий, существующих между гидродинамикой, геометрической оптикой, механикой и термодинамикой, можно найти в книге [16].

\section{5. Вихревая теория волчка Эйлера}

Ключевой вопрос применимости общей теории вихрей из 4 состоит в нахождении инвариантных многообразий, однозначно проектирующихся на конфигурационное пространство. Этот вопрос легко и естественно решается для волчка Эйлера [17].

Пусть $\alpha, \beta, \gamma$ - ортонормированный репер в неподвижном пространстве. Мы будем рассматривать их как векторы в подвижном пространстве, связанном с твердым телом. Тогда они уже не будут постоянными; их эволюция со временем описывается уравнениями Пуассона

$$
\dot{\alpha}+\omega \times \alpha=0, \quad \dot{\beta}+\omega \times \beta=0, \quad \dot{\gamma}+\omega \times \gamma=0 .
$$


Эти уравнения вместе с динамическими уравнениями Эйлера (8) образуют полную систему уравнений движения (в предположении, что момент сил $M$, действующих на тело, - известная функция от положения и угловой скорости твердого тела). В случае $M=0$ эти уравнения допускают следующие интегралы:

$$
(I \omega, \alpha)=c_{1}, \quad(I \omega, \beta)=c_{2}, \quad(I \omega, \gamma)=c_{3},
$$

выражающие свойство неизменности вектора кинетического момента тела $K=I \omega$ как вектора в неподвижном пространстве.

Интегралы (19) имеют прозрачную групповую интерпретацию. Конфигурационное пространство волчка, вращающегося вокруг неподвижной точки, - это группа поворотов трехмерного евклидова пространства $\mathrm{SO}(3)$. Вращениям волчка с постоянной угловой скоростью $\omega=\alpha$ отвечает правоинвариантное векторное поле скоростей на $\mathrm{SO}(3)$. Фазовый поток этого поля состоит, очевидно, из левых сдвигов на группе $\mathrm{SO}(3)$. Однако кинетическая энергия волчка $T=(I \omega, \omega) / 2$ инвариантна при левых сдвигах. Следовательно, по теореме Нётер, уравнения движения допускают интеграл

$$
\left(\frac{\partial T}{\partial \omega}, \alpha\right)=(I \omega, \alpha)=\text { const. }
$$

Эти соображения фактически содержатся в краткой заметке А. Пуанкаре [18]. Там же введены более общие системы на группах Ли с левоинвариантной метрикой (кинетической энергией), о которых будет идти речь ниже.

Из формул (19) вытекает очевидное равенство

$$
I \omega=c_{1} \alpha+c_{2} \beta+c_{3} \gamma
$$

Оно позволяет представить скорость вращения волчка как однозначную функцию на конфигурационном пространстве. Другими словами, векторное равенство (20) задает трехпараметрическое семейство трехмерных стационарных инвариантных многообразий, однозначно проектирующихся на конфигурационное пространство $M=\mathrm{SO}(3)$. В дальнейшем рассматривается нетривиальный случай, когда $c_{1}^{2}+c_{2}^{2}+c_{3}^{2} \neq 0$.

Для упрощения формул свяжем с твердым телом трехгранник, образованный главными осями инерции тела относительно точки закрепления. В этих осях тензор инерции приводится к диагональному виду: $I=\operatorname{diag}\left(I_{1}, I_{2}, I_{3}\right)$. Пусть $\omega_{1}, \omega_{2}, \omega_{3}-$ проекции 
вектора угловой скорости $\omega$ на эти подвижные оси. Тогда вид кинетической энергии упрощается:

$$
T=\frac{1}{2}\left(I_{1} \omega_{1}^{2}+I_{2} \omega_{2}^{2}+I_{3} \omega_{3}^{2}\right) .
$$

Для того чтобы представить инвариантные многообразия (19) в канонических переменных, введем в качестве обобщенных координат на группе $\mathrm{SO}(3)$ известные углъ Эйлера $\vartheta, \varphi, \psi$. Они однозначно определяют положение главных осей инерции твердого тела относительно неподвижного трехгранника. Угловые скорости выражаются через углы Эйлера и их производные с помощью кинематических формул Эйлера (1760):

$$
\begin{aligned}
\omega_{1} & =\dot{\psi} \sin \vartheta \sin \varphi+\dot{\theta} \cos \varphi, \\
\omega_{2} & =\dot{\psi} \sin \vartheta \cos \varphi-\dot{\theta} \sin \varphi, \\
\omega_{3} & =\dot{\psi} \cos \vartheta+\dot{\varphi}
\end{aligned}
$$

Они позволяют представить кинетическую энергию (21) как положительно определенную квадратичную форму по обобщенным скоростям $\dot{\vartheta}, \dot{\varphi}, \dot{\psi}$. Сопряженные канонические импульсы вводим по обычному правилу:

$$
p_{\psi}=\frac{\partial T}{\partial \dot{\psi}}, \quad p_{\theta}=\frac{\partial T}{\partial \dot{\theta}}, \quad p_{\varphi}=\frac{\partial T}{\partial \dot{\varphi}} .
$$

Используя (21) и (22), получим следующие формулы:

$$
\begin{aligned}
p_{\psi} & =I_{1} \omega_{1} \frac{\partial \omega_{1}}{\partial \dot{\psi}}+I_{2} \omega_{2} \frac{\partial \omega_{2}}{\partial \dot{\psi}}+I_{3} \omega_{3} \frac{\partial \omega_{3}}{\partial \dot{\psi}} \\
& =I_{1} \omega_{1} \sin \theta \sin \varphi+I_{2} \omega_{2} \sin \vartheta \cos \varphi+I_{3} \omega_{3} \cos \theta, \\
p_{\theta} & =I_{1} \omega_{1} \cos \varphi-I_{2} \omega_{2} \sin \varphi, \\
p_{\varphi} & =I_{3} \omega_{3} .
\end{aligned}
$$

С учетом формулы (20) и известных выражений компонент векторов $\alpha, \beta, \gamma$ через углы Эйлера получаем окончательный вид инвариантных многообразий волчка Эйлера в канонических переменных:

$$
\begin{aligned}
& p_{\psi}=c_{3} \\
& p_{\vartheta}=c_{1} \cos \psi+c_{2} \sin \psi \\
& p_{\varphi}=c_{1} \sin \vartheta \sin \psi-c_{2} \sin \vartheta \cos \psi+c_{3} \cos \vartheta
\end{aligned}
$$


Интересно отметить, что эти формулы не зависят от моментов инерции тела $I_{1}, I_{2}, I_{3}$.

Поскольку на волчок не действуют никакие силы, то неподвижный репер $\alpha, \beta, \gamma$ мы можем выбирать произвольно. Направим, например, вектор $\gamma$ вдоль постоянного в пространстве вектора кинетического момента $K=I \omega$. Тогда, очевидно,

$$
c_{1}=c_{2}=0, \quad c_{3}=k, \quad k=|K| .
$$

При фиксированном $k$ инвариантные многообразия (23) задают на группе $\mathrm{SO}(3)$ динамическую систему

$$
\dot{x}=v(x), \quad x=(\psi, \vartheta, \varphi) .
$$

С использованием кинематических формул Эйлера эти уравнения легко записать в явном виде:

$$
\begin{aligned}
& \dot{\psi}=k\left(\frac{\sin ^{2} \varphi}{I_{1}}+\frac{\cos ^{2} \varphi}{I_{2}}\right), \\
& \dot{\vartheta}=k\left(\frac{1}{I_{1}}-\frac{1}{I_{2}}\right) \sin \vartheta \sin \varphi \cos \varphi, \\
& \dot{\varphi}=k \cos \theta\left(\frac{1}{I_{3}}-\frac{\sin ^{2} \varphi}{I_{1}}-\frac{\cos ^{2} \varphi}{I_{2}}\right) .
\end{aligned}
$$

Их фазовый поток задает стационарное "течение" воображаемой жидкости на группе $\mathrm{SO}(3)$. Опишем его основные свойства.

$1^{\circ}$. "Внутренняя" метрика на $\mathrm{SO}(3)$, задаваемая кинетической энергией волчка, позволяет вычислить ротор векторного поля $v$ (поля (24)). Это поле - вихревое. Рассматриваемое как поле скоростей, оно порождает вращение твердого тела с угловой скоростью $\omega=\mu \gamma, \mu=$ const и коммутирует с полем $v$. В частности, поле $\operatorname{rot} v$ правоинвариантно и все вихревые линии замкнуты. Расслоение группы $\mathrm{SO}(3)$ вихревыми линиями совпадает с известным в топологии расслоением Хопфа.

$2^{\circ}$. Уравнения $(24)$ допускают интегральный инвариант

$$
\iiint \sin \vartheta d \psi d \theta d \varphi, \quad 0 \leqslant \vartheta \leqslant \pi
$$

Он задает на группе $\mathrm{SO}(3)$ меру, инвариантную относительно левых и правых сдвигов. 
Этот факт позволяет говорить о сохранении “массы” воображаемой жидкости на группе $\mathrm{SO}(3)$. Ее плотность совпадает с плотностью биинвариантной меры (единственной с точностью до постоянного множителя).

$3^{\circ}$. Интеграл Бернулли $h$ равен

$$
\frac{1}{2} k^{2}\left(I^{-1} \gamma, \gamma\right)
$$

Критические точки функции $h$ - орбиты постоянных вращений волчка вокруг главных осей инерции (с фиксированным значением кинетического момента $K$ ), а критические значения совпадают со значениями энергии на этих вращениях. Если $c$ не является критическим значением функции $h$, то поверхность Бернулли

$$
B_{c}=\{x \in \mathrm{SO}(3): h(x)=c\}
$$

- двумерный тор. Векторные поля $v$ и $\operatorname{rot} v$ касаются $B_{c}$, коммутируют и линейно независимы в каждой точке. В частности, движение по этому тору будет условно-периодическим.

Эти результаты переносятся (с естественными изменениями) на общий случай систем на группах Ли с левоинвариантной кинетической энергией. Пусть $G$ - связная группа Ли - конфигурационное пространство механической системы, а $T(\dot{x}, x)$ - кинетическая энергия, инвариантная относительно всех левых сдвигов на группе $G$.

Пусть $w_{1}, \ldots, w_{n}$ - базис правоинвариантных векторных полей на $G$. Их фазовые потоки - семейства левых сдвигов на группе $G$. Поэтому (по теореме Нётер) уравнения движения допускают $n$ независимых первых интегралов

$$
\frac{\partial T}{\partial \dot{x}} \cdot w_{1}=c_{1}, \quad \ldots, \quad \frac{\partial T}{\partial \dot{x}} \cdot w_{n}=c_{n},
$$

линейных относительно скорости $\dot{x}$. Из этих соотношений можно выразить скорость системы в виде однозначной функции от положения $x$ и параметров $c=\left(c_{1}, \ldots, c_{n}\right)$ :

$$
\dot{x}=v_{c}(x), \quad x \in G .
$$

Напомним, что на каждой группе Ли имеется единственная (с точностью до постоянного множителя) мера, инвариантная при всех левых (правых) сдвигах. В случае унимодулярной группы эта мера (называемая мерой Хаара) биинвариантна. В частности, все компактные группы унимодулярны. 
Tеорема 2 [19]. При фиксированном значении $c \in \mathbb{R}^{n}$ фазовый поток системы (25) сохраняет правоинвариантную меру на $G$.

СлЕДСТвИЕ. Если группа $G$ унимодулярная, то фазовый поток системы (25) сохраняет меру Хаара на $G$.

Это утверждение обобщает свойство $2^{\circ}$ волчка Эйлера.

Развиваемый подход можно применить и к бесконечномерной группе диффеоморфизмов гладкого многообразия $Q$, сохраняющих элемент объема. Чтобы привязать последующие построения к обычной гидродинамике идеальной жидкости (скажем, с периодическими граничными условиями), в качестве $Q$ можно взять, например, трехмерный тор с "плоской" метрикой и угловыми координатами $x=\left(x_{1}, x_{2}, x_{3}\right)$. Эта группа обычно обозначается $\operatorname{SDiff} Q$. Алгебра Ли группы SDiff $Q$ состоит из касательных векторных полей на $Q$ с нулевой дивергенцией. Определим скалярное произведение двух элементов этой алгебры (т. е. двух соленоидальных векторных полей $v_{1}$ и $v_{2}$ ) с помощью формулы

$$
\left\langle v_{1}, v_{2}\right\rangle=\int\left(v_{1}, v_{2}\right) d^{3} x
$$

Рассмотрим теперь течение однородной идеальной жидкости в области $Q$; для простоты будем считать плотность жидкости равной единице. Уравнение неразрывности приводит к условию несжимаемости: $\operatorname{div} v=0$. Течения жидкости описываются кривыми $g^{t}$ на группе SDiff $Q$ : диффеоморфизм $g^{t}: Q \rightarrow Q$ переводит каждую частицу из ее начального положения в положение, которое она занимает в момент времени $t$.

Несложно проверить, что кинетическая энергия жидкости

$$
T=\frac{1}{2}\langle v, v\rangle
$$

- правоинвариантная риманова метрика на группе $\operatorname{SDiff} Q$. Свойство правоинвариантности отличает эту систему от волчка Эйлера и создает дополнительные трудности при ее изучении.

В 1960-е годы было подмечено следующее важное обстоятельство: течения идеальной несжимаемой жидкости - геодезические линии метрики (26). Таким образом, идеальная несжимаемая жидкость - это бесконечномерный “волчок Эйлера" с правоинвариантной метрикой на группе $\operatorname{SDiff} Q$ [20]-[22]. Это - следствие 
принципа наименьшего действия, который, если угодно, можно считать определением динамики идеальной жидкости.

Как известно, правые сдвиги на группе Ли включаются в фазовые потоки левоинвариантных полей. Оказывается, левоинвариантные поля $u(x, t)$ на группе $\operatorname{SDiff} Q$ - это соленоидальные поля на $Q$, удовлетворяющие уравнению

$$
\frac{\partial u}{\partial t}=\operatorname{rot}(v \times u) .
$$

Оно выражает свойство вмороженности интегральных кривых поля $u$ в течение жидкости. С учетом несжимаемости уравнение (27) эквивалентно "уравнению Эйлера"

$$
\frac{\partial u}{\partial t}=[v, u]
$$

где $[\cdot, \cdot]$ - коммутатор векторных полей. Следовательно, снова по теореме Нётер, уравнения геодезических на группе SDiff $Q$ допускают бесконечную серию линейных законов сохранения:

$$
\langle u, v\rangle=\int_{Q}(u, v) d^{3} x=\text { const. }
$$

Это утверждение (установленное в [16, доп. 1]) обобщает старую теорему Ж.-Ж. Моро, в которой $u=\operatorname{rot} v$.

Поскольку кинетическая энергия (26) представляет собой невырожденную квадратичную форму, то наличие бесконечной серии интегралов (28) позволяет надеяться найти скорость течения $v$ как функцию на группе SDiff $Q$. Отличие от левоинвариантного случая состоит в том, что фигурирующие в (28) векторные поля $u$ не заданы априори, а их надо искать как решения уравнения (27). Если эту задачу считать решенной, то на группе SDiff $Q$ естественным образом возникает бесконечномерная динамическая система, фазовый поток которой схож по своим свойствам со стационарным течением невязкой жидкости. Было бы интересным изучить эту систему с гидродинамической точки зрения, изложенной в 4 (вихревые векторы и многообразия, поверхности Бернулли, инвариантные меры, ... ). Такой подход можно назвать вторичной гидродинамикой. Первые (но далеко не исчерпывающие) шаги в этом направлении сделаны в работе [23]. 


\section{6. Энергетические критерии устойчивости}

Как уже было сказано, Эйлер впервые изучал свойства упругих систем мало отклоняться от состояния равновесия при малых возмущающих воздействиях. Современная теория устойчивости упругих систем основана на распространении классической теории устойчивости на континуальные системы и может рассматриваться как часть теории дифференциальных уравнений в гильбертовом пространстве.

Для отыскания условий устойчивости упругих систем в потенциальном силовом поле обычно используют энергетический подxод, основанный на теореме Лагранжа-Дирихле: положение равновесия устойчиво, если оно является точкой строгого локального минимума потенциальной энергии. Эта энергия - функционал от поля перемещений частиц упругой системы, зависящий еще от некоторых параметров (нагрузка, длина стержня, жесткость при изгибе и т.д.). Область устойчивости в пространстве параметров определяется условием, что вторая вариация потенциальной энергии - положительно определенная квадратичная форма. На границе области устойчивости эта форма становится вырожденной и, как правило, происходит бифуркация положений равновесия.

На практике широко применяют аппроксимацию континуальных систем конечномерными системами. Еще Лагранж заменял упругую среду набором большого числа малых частиц, упруго взаимодействующих между собой. Кстати сказать, с физической точки зрения такая модель даже ближе к действительности. В соответствии с этим замечанием мы обсудим некоторые новые идеи исследования устойчивости тривиального решения линейной системы

$$
\dot{x}=A x, \quad x \in \mathbb{R}^{n},
$$

допускающей квадратичный первый интеграл

$$
f=\frac{1}{2}(B x, x) .
$$

Матрицы $A$ и $B$ ( $B$ симметрична) будем считать невырожденными. Практически все, сказанное ниже, можно перенести на бесконечномерный случай с необходимыми предосторожностями.

Поскольку система (29) линейная, то устойчивость по Ляпунову положения равновесия $x=0$ означает ограниченность всех ее 
решений. Квадратичный интеграл (30) можно интерпретировать как интеграл энергии. Конечно, критерий устойчивости равновесия $x=0$ можно сформулировать через спектральные свойства матрицы $A$. Однако, имея в виду энергетический подход, полезно связать свойство устойчивости также и со свойствами квадратичного интеграла (30) (например, с его сигнатурой).

Теорема 3 [24]. Справедливы следующие заключения:

1) $п$ четно ;

2) спектр матрицы А симметричен относительно вещественной и мнимой осей;

3) $\operatorname{div}(A x)=\operatorname{tr} A=0$;

4) если индекс инериии квадратичной формы (30) нечетный, то равновесие $x=0$ неустойчиво;

5) система (29) имеет $n / 2$ независимых квадратичных интегралов;

6) равновесие $x=0$ устойчиво в том и только том случае, когда (29) допускает положительно определенный квадратичный интеграл.

Всеми этими свойствами обладают линейные гамильтоновы системы. Оказывается, это замечание не случайно: система (29) в действительности гамильтонова, причем $f$ - функция Гамильтона. Только эта система представлена, вообще говоря, не в канонической форме. Соответствующая симплектическая структура задается билинейной формой

$$
\omega\left(x^{\prime}, x^{\prime \prime}\right)=\left(\Omega x^{\prime}, x^{\prime \prime}\right), \quad \Omega=B A^{-1} .
$$

Действительно, эта билинейная форма, очевидно, невырождена. Надо показать, что $\Omega$ - кососимметрическая матрица. Для доказательства воспользуемся равенством $A^{T} B=-B A$, которое выражает свойство квадратичной формы (30) быть первым интегралом системы (29). Итак,

$$
A^{T}=-B A B^{-1}, \quad\left(A^{T}\right)^{-1}=-B A^{-1} B^{-1} .
$$

Поэтому

$$
\Omega^{T}=\left(A^{T}\right)^{-1} B=-\left(B A^{-1} B^{-1}\right) B=-B A^{-1}=-\Omega .
$$

После этого наблюдения систему (29) уже совсем легко представить в гамильтоновой форме:

$$
\omega(\dot{x}, \cdot)=(\Omega \dot{x}, \cdot)=(B x, \cdot)=d f(\cdot) .
$$


Заключение 4) теоремы 3 обобщает классическую теорему Томсона о невозможности гироскопической стабилизации равновесия с нечетной степенью неустойчивости Пуанкаре (с нечетным индексом инерции потенциальной энергии). Его можно представить в несколько иной форме, если ввести степень неустойчивости и системы (29) как количество собственных значений матрицы $A$ в правой комплексной полуплоскости. Пусть $i^{-}\left(i^{+}\right)-$ отрицательный (положительный) индекс инерции квадратичной формы $f$. Тогда

$$
u \equiv i^{-}(\bmod 2) .
$$

Так как $i^{-}+i^{+}=n$ и $n$ четно, то в этом сравнении $i^{-}$, конечно, можно заменить на $i^{+}$.

Однако, как показано в [25], сравнение (31) справедливо и в более общем случае, когда

$$
\dot{f}=(B A x, x) \leqslant 0 .
$$

Здесь $n$ уже может быть и нечетным. Этот случай отвечает системам с диссипацией энергии. Если диссипация полная (квадратичная форма (32) отрицательно определена), то $u=i^{-}$(теорема Островского-Шнейдера [26]).

Обобщенную теорему Томсона (31) можно распространить и на некоторые случаи, когда матрица $A$ вырождена. Вектор $x \in \mathbb{R}^{n}$ назовем изотропным, если $(B x, x)=0$. Конечно, если форма $f$ положительно или отрицательно определена, то изотропным будет только нулевой вектор.

Tеорема 4 [27]. Пусть $n$ четно, $|B| \neq 0, i^{-}\left(i^{+}\right)$нечетно u $A x \neq 0$ для всех изотропных $x \neq 0$. Тогда матрица $A$ имеет пару ненулевых вещественных чисел $\pm \lambda$ с изотропными собственными векторами.

Более содержательным и сложным выглядит вопрос об оценке степени устойчивости $s$ : это количество пар чисто мнимых собственных значений матрицы $A$. Снова считаем $A$ и $B$ невырожденными матрицами.

Tеорема 5 [28]. Справедлива оценка

$$
\left|i^{+}-i^{-}\right| \leqslant 2 l,
$$

где $l$ - количество пар чисто мнимых собственных чисел матрицы $A$ с жордановыми клетками нечетного порядка. 
СлеДСтвиЕ 1. Справедлива ощенка

$$
\left|i^{+}-i^{-}\right| \leqslant 2 s .
$$

СлЕДСТвИЕ 2. Пусть индекс $i^{-}\left(\right.$или $\left.i^{+}\right)$равен единице. Тогда спектр А состоит из пары вещественных ненулевых чисел $\pm \lambda u(n-2) / 2$ пар чисто мнимых собственных значений с простыми элементарными делителями.

Последнее утверждение описывает механизм потери устойчивости в типичном случае, когда теряется положительная определенность полной энергии. Это имеет прямое отношение и к рассмотренным Эйлером задачам о потере устойчивости равновесий упругих стержней.

Пусть $2 i^{-} \leqslant n$. Тогда, с учетом соотношений $i^{+}+i^{-}=n$ и $u+s=n / 2$, неравенство (33) эквивалентно неравенству

$$
u \leqslant i^{-} .
$$

В таком виде оно было установлено ранее в работах [29], [30] для гамильтоновых систем частного вида, описывающих линейную динамику механических систем в потенциальном поле с дополнительными гироскопическими силами. В работе А. А. Шкаликова [31] содержится уточнение неравенства (34), основанное на обобщении теоремы Понтрягина о самосопряженных операторах в пространствах с индефинитной метрикой. Наш подход, применяемый к линейным гамильтоновым системам общего вида, использует теорию нормальных форм Вильямсона.

\section{7. Задача двух центров в пространствах постоянной кривизны}

Оказывается, классический результат Эйлера об интегрируемости задачи двух неподвижных центров допускает любопытное обобщение на пространства постоянной кривизны. Но сначала надо дать пояснения о гравитации в этих пространствах.

Хорошо известно, что потенциал гравитационного взаимодействия в обычном трехмерном евклидовом пространстве обладает двумя фундаментальными свойствами. С одной стороны, это гармоническая функция (зависит лишь от расстояния и удовлетворяет уравнению Лапласа), а с другой стороны только этот 
потенциал и еще потенциал упругой пружины порождают центральные силовые поля, в которых все ограниченные орбиты частицы замкнуты (теорема Бертрана). Эти свойства естественным образом переносятся на более общий случай трехмерных пространств постоянной кривизны (трехмерная сфера $S^{3}$ и пространство Лобачевского $\left.L^{3}\right)$.

Для определенности записи рассмотрим трехмерную сферу. Пусть материальная частица $m$ единичной массы движется в поле сил с потенциалом $V$, зависящим лишь от расстояния между этой частицей и фиксированной точкой $M \in S^{3}$. Эта задача - аналог классической задачи о движении в центральном поле. Пусть $\vartheta-$ длина дуги большого круга, соединяющего точки $m$ и $M$, измеряемая в радианах. Тогда $V$ зависит только от угла $\vartheta$. Для определения гравитационного потенциала на сфере уравнение Лапласа следует заменить уравнением Лапласа-Бельтрами:

$$
\Delta V=\sin ^{-2} \vartheta \cdot \frac{\partial}{\partial \theta}\left(\sin ^{2} \theta \cdot \frac{\partial V}{\partial \vartheta}\right)=0 .
$$

Оно сразу же решается:

$$
V=-\gamma \operatorname{ctg} \theta+\alpha, \quad \alpha, \gamma=\text { const. }
$$

Аддитивная константа $\alpha$ несущественна; положим далее $\alpha=0$. Для определенности рассмотрим случай $\gamma>0$ (тогда точка $M$ будет притягивать точку $m$ ). Параметр $\gamma$ играет роль гравитационной постоянной, умноженной на массу притягивающего центра $M$. В дополнение к притягивающему центру $M$ это силовое поле имеет отталкивающий центр $M^{\prime}$ в антиподальной точке (где $\vartheta=\pi)$.

В общем случае, когда $V$ - произвольная функция от угла $\vartheta$, траектории точки $m$ лежат на двумерных сферах $S^{2}$, содержащих точки $M$ и $M^{\prime}$.

Естественно рассмотреть также обобщенную задачу Бертрана: среди всех потенциалов $V(\vartheta)$ выделить те, в поле которых почти все орбиты точки $m$ на двумерной сфере $S^{2}$ замкнуты. Ее решением (как и в классическом случае) являются два потенциала:

$$
V_{1}=-\gamma \operatorname{ctg} \vartheta, \quad V_{2}=\frac{k}{2} \operatorname{tg}^{2} \theta, \quad \gamma, k=\text { const } .
$$

Первый из них - гравитационный потенциал, а второй - аналог потенциал Гука ( $k$ - “коэффициент упругости"). 
Движение частицы в центральном гравитационном поле подчиняется обобщенным законам Кеплера. В частности, ее орбиты квадрики на $S^{2}$ - линии пересечения сферы с конусом второго порядка, вершина которого совпадает с центром сферы.

Все эти результаты (с различных точек зрения и в разной общности) получены рядом авторов, зачастую не знавших о существовании работ предшественников. По-видимому, первой (и очень обстоятельной) работой на эту тему была забытая статья известного немецкого геометра Вильгельма Киллинга. Эти работы собраны и систематизированы А. В. Борисовым и И. С. Мамаевым в сборнике [32].

Рассмотрим теперь обобщенную задачу двух неподвижных центров. Речь идет о движении точки по двумерной сфере в поле с потенциалом $V_{1}+V_{2}$, где

$$
V_{1}=\gamma_{1} \operatorname{ctg} \vartheta_{1}, \quad V_{2}=\gamma_{2} \operatorname{ctg} \vartheta_{2}, \quad \gamma_{1}, \gamma_{2}=\text { const. }
$$

Здесь $\vartheta_{1}, \vartheta_{2}$ - дуги большого круга, проходящие через неподвижные гравитирующие центры $M_{1}, M_{2}$ и движущуюся частицу.

Уравнения движения в форме Гамильтона решаются разделением переменных в ортогональных сфероконических координатах на $S^{2}$. Это - результат Киллинга. Наше наблюдение состоит в том, что задача останется вполне интегрируемой, если добавить упругую силу (с обобщенным гуковским потенциалом) с центром, лежащим ровно посредине между $M_{1}$ и $M_{2}$ [33]. Этот результат вполне аналогичен добавлению Лагранжа к классической задаче Эйлера. Естественно, что все сказанное справедливо и в пространстве Лобачевского.

Затронутое выше - это небольшая часть творческого наследия Леонарда Эйлера. А ведь мы не касались его выдающихся результатов в анализе, геометрии и теории чисел! Осознавая все это, закончим известным призывом Лапласа: “Читайте Эйлера, изучайте Эйлера. Он наш общий учитель!" 


\section{Список литературы}

[1] А.Н. Крылов, “Леонард Эйлер", Собрание трудов академика А.Н. Крылова. Т. 1. Ч. 2. Научно-популярные статъи. Биографические характеристики, Изд-во АН СССР, М.-Л., 1951, 192-217.

[2] Г. Харди, Расходящиеся ряды, ИЛ, М., 1951 MR 0067217; пер. с англ.: G.H. Hardy, Divergent series, Clarendon, Oxford, 1949 MR 0030620, Zbl 0032.05801.

[3] А.Н. Кузнецов, "Дифференцируемые решения вырождающихся систем обыкновенных уравнений”, Функи. анализ и его прилож., 6:2 (1972), 41-51 Mi faa2494, MR 315189, Zbl 0259.34005; англ. пер.: A.N. Kuznetsov, "Differentiable solutions to degenerate systems of ordinary equations", Funct. Anal. Appl., 6:2 (1972), 119127 doi $10.1007 /$ BF01077515.

[4] А.Н. Кузнецов, "О существовании входящих в особую точку решений автономной системы, обладающей формальным решением", Функи. анализ и его прилож., 23:4 (1989), 63-74 Mi faa1068, MR 1035375, Zbl 0717.34004; англ. пер.: A. N. Kuznetsov, "Existence of solutions entering at a singular point of an autonomous system having a formal solution", Funct. Anal. Appl., 23:4 (1989), 308-317 doi 10.1007/BF01078945.

[5] Ж.-П. Рамис, Расходящиеся ряды и асимптотическая теория, Ин-т компьютерных исследований, Москва-Ижевск, 2002; пер. с франц.: J.-P. Ramis, Séries divergentes et théories asymptotiques, Panoramas et Synthèses, 121, Soc. Math. France, Paris, 1993 MR 1272100, Zbl 0830.34045.

[6] В.В. Козлов, "Асимптотические движения и проблема обращения теоремы Лагранжа-Дирихле", ПММ, 50:6 (1986), 928-937 MR 922185, Zbl 0631.70018, ADS 1986PriMM. .50..928K; англ. пер.: V. V. Kozlov, "Asymptotic motions and the inversion of the LagrangeDirichlet theorem", J. Appl. Math. Mech., 50:6 (1986), 719-725 doi 10.1016/0021-8928 (86)90079-1.

[7] В.В. Козлов, В.П. Паламодов, “Об асимптотических решениях уравнений классической механики”, Докл. АН CCCP, 263:2 (1982), 285-289 MR 650153, Zbl 0504.70018; англ. пер.: V. V. Kozlov, V.P. Palamodov, "On asymptotic solutions of the equations of classical mechanics", Soviet Math. Dokl., 25:2 (1982), 335-339.

[8] В.В. Козлов, С. Д. Фурта, Асимптотики решений сильно нелинейных систем дифференииальных уравнений, Изд-во МГУ, М., 1996 MR 1457796, Zbl 0949.34003.

[9] К.Г.Я. Якоби, Лекиии по аналитической механике, Ин-т компьютерных исследований, Москва-Ижевск, 2006; пер. с нем.: C. G. J. Jacobi, Vorlesungen über analytische Mechanik, Dokumente 
Gesch. Math., 8, Deutsche Mathematiker Vereinigung, Freiburg, 1996 MR 1414679, Zbl 0867.01051.

[10] В.И. Арнольд, "О топологии трехмерных стационарных течений идеальной жидкости", ПММ, 30:1 (1966), 183-185 MR 0235578, Zbl 0156.23002; англ. пер.: V. I. Arnol'd, "On the topology of threedimensional steady flows of an ideal fluid", J. Appl. Math. Mech., 30:1 (1966), 223-226 doi 10.1016/0021-8928(66)90070-0.

[11] В.В. Козлов, "Замечания о стационарных движениях сплошной среды", ПММ, 47:2 (1983), 341-342 MR 740637, Zbl 0564.76116, ADS 1983PriMM. .47 . .341K; англ. пер.: V. V. Kozlov, "Notes on steady vortex motions of continuous medium", J. Appl. Math. Mech., 47:2 (1983), 288-289 doi 10.1016/0021-8928(83)90020-5.

[12] С.В. Болотин, "Неинтегрируемость задачи $n$ центров при $n>$ 2", Вестн. Моск. ун-та. Сер. 1. Матем., мех., 1984, №3, 65-68 MR 749024, Zbl 0551.70008; англ. пер.: S. V. Bolotin, "Nonintegrability of the $N$-center problem for $N>2$ ", Mosc. Univ. Mech. Bull., 39:3 (1984), 24-28 Zbl 0653.70016.

[13] В.В. Козлов, "Гидродинамика гамильтоновых систем", Вестн. Моск. ун-та. Сер. 1. Матем., мех., 1983, № 6, 10-22 MR 0728549, Zbl 0552.76006; англ. пер.: V. V. Kozlov, "The hydrodynamics of Hamiltonian systems", Mosc. Univ. Mech. Bull., 38:6 (1983), 9-23 $\mathrm{Zbl} 0585.76004$.

[14] В.В. Козлов, "Об одном обобщении метода ГамильтонаЯкоби", ПММ, 60:6 (1996), 929-939 MR 1627715, Zbl 1040.70503; англ. пер.: V.V. Kozlov, "An extension of the HamiltonJacobi method", J. Appl. Math. Mech., 60:6 (1996), 911-920 doi 10.1016/S0021-8928(96)00113-X.

[15] И. С. Аржаных, Поле импулъсов, Наука, Ташкент, 1965 мR 0203980; англ. пер.: I.S. Arzhanykh, Momentum fields, Nat. Lending Lib., Boston Spa, Yorkshire, 1971 Zbl 0225.70001.

[16] В. В. Козлов, Общая теория вихрей, Удмурт. ун-т, Ижевск, 1998 MR 1707302, Zbl 1054.37514; англ. пер.: V. V. Kozlov, Dynamical systems. X. General theory of vortices, Encyclopaedia Math. Sci., 67, Springer-Verlag, Berlin, 2003 MR 1995646, Zbl 1104.37050.

[17] В.В. Козлов, "Вихревая теория волчка", Вестн. Моск. ун-та. Сер. 1. Матем., мех., 1990, № 4, 56-62 MR 1086606, Zbl 0850.70068; англ. пер.: V. V. Kozlov, "Eddy theory of the top", Mosc. Univ. Mech. Bull., 45:4 (1990), 26-38.

[18] H. Poincaré, "Sur une forme nouvelle des équations de la méchanique", C. R. Acad. Sci. Paris, 132 (1901), 369-371 Zbl 32.0715.01.

[19] В.В. Козлов, В.А. Ярощук, "Об инвариантных мерах уравнений Эйлера-Пуанкаре на унимодулярных группах", Вестн. Моск. ун-та. Сер. 1. Матем., мех., 1993, № 2, 91-95 MR 1223989, 
Zbl 0804.58034; англ. пер.: V. V. Kozlov, V. A. Yaroshchuk, "On the invariant measures of Euler-Poincaré equations on unimodular groups", Mosc. Univ. Mech. Bull., 48:2 (1993), 45-50.

[20] J.-J. Moreau, "Une méthode de "cinématique fonctionnelle" en hydrodynamique", C.R. Acad. Sci. Paris, 249 (1959), 2156-2158 MR 122197, Zbl 0117.19601.

[21] В.И. Юдович, "Плоские нестационарные движения идеальной несжимаемой жидкости", Докл. АН СССР, 136:3 (1961), 564-567 Zbl 0098.39602; англ. пер.: V. I. Yudovich, "Plane unsteady motion of an ideal incompressible fluid", Soviet Phys. Dokl., 6 (1961), 18-20 ADS 1961SPhD ...6. . . 18Y.

[22] V.I. Arnol'd, "Sur un principe variationnel pour les écoulements stationnaires des liquides parfaits et ses applications aux problèmes de stabilité non linéaires", J. Mécanique, 5:1 (1966), 29-43 Zbl 0161.22903.

[23] М. В. Дерябин, Ю. Н. Федоров, "О редукциях на группу геодезических потоков с (лево-) правоинвариантной метрикой и их полях симметрий", Докл. РАН, 391:4 (2003), 439-442 MR 2043032; англ. пер.: M. V. Deryabin, Yu. N. Fedorov, "On reductions for a group of geodesic flows with (left-) right-invariant metric, and their symmetry fields", Dokl. Math., 68:1 (2003), 75-78.

[24] В.В. Козлов, "Линейные системы с квадратичным интегралом", ПММ, 56:6 (1992), 900-906 MR 1229017, Zbl 0792.70014; англ. пер.: V.V. Kozlov, "Linear systems with a quadratic integral", J. Appl. Math. Mech., 56:6 (1992), 803-809 doi 10.1016/0021-8928(92) 90114-N.

[25] В. В. Козлов, "О степени неустойчивости", ПММ, 57:5 (1993), 1419 MR 1262057, Zbl 0798.70013; англ. пер.: V. V. Kozlov, "On the degree of instability", J. Appl. Math. Mech., 57:5 (1993), 771-776 doi 10.1016/0021-8928(93)90141-8.

[26] A. Ostrowski, H. Schneider, "Some theorems on the inertia of general matrices", J. Math. Anal. Appl., 4:1 (1962), 72-84 doi 10.1016/0022-247X (62) 90030-6, MR 0142555, Zbl 0112.01401.

[27] В.В. Козлов, “Замечания о собственных числах вещественных матриц", Докл. РАН, 403:5 (2005), 589-592 MR 2215319; англ. пер.: V. V. Kozlov, "Remarks on eigenvalues of real matrices", Dokl. Math., 72:1 (2005), 567-569.

[28] В.В. Козлов, А.А. Карапетян, "О степени устойчивости", Дифферени. уравнения, 41:2 (2005), 186-192 MR 2202018, Zbl 1090.34564; англ. пер.: V. V. Kozlov, A. A. Karapetyan, "On the degree of stability", Differ. Equ., 41:2 (2005), 195-201 doi 10.1007/s10625-005-0149-7.

[29] H. K. Wimmer, "Inertia theorems for matricies, controllability, and linear vibrations", Linear Algebra Appl., 8:4 (1974), 337-343 doi 10.1016/0024-3795(74)90060-3, MR 394388, Zbl 0288.15015. 
[30] P. Lancaster, M. Tismenetsky, "Inertia characteristics of selfajoint matrix polynomials", Linear Algebra Appl., 52-53 (1983), 479-496 doi 10.1016/0024-3795 (83)80030-5, MR 709367, Zbl 0516.15018.

[31] A. A. Shkalikov, "Operator pencils arising in elasticity and hydrodynamics: the instability index formula", Recent developments in operator theory and its applications (Winnipeg, Canada, 1994), Oper. Theory Adv. Appl., 87, Birkhäuser, Basel, 1996, 358-385 MR 1399370, Zbl 0860.47009.

[32] А.В. Борисов, И.С. Мамаев (ред.), Классическая динамика в неевклидовых пространствах, Ин-т компьютерных исследований, Москва-Ижевск, 2004.

[33] V. V. Kozlov, A. O. Harin, "Kepler's problem in constant curvature spaces", Celestial Mech. Dynam. Astronom., 54:4 (1992), 393-399 doi 10.1007/BF00049149, MR 1188291, Zbl 0765.70007, ADS 1992CeMDA . .54. .393K. 